\title{
Investigation on Relation between Index of Difficulty in Fitts' Law and Device Screen Sizes
}

\author{
Hidehiko Okada $^{1}$, Takayuki Akiba ${ }^{1}$, and Ryosuke Fujioka ${ }^{2}$ \\ ${ }^{1}$ Kyoto Sangyo University \\ Kamigamo Motoyama, Kita-ku, Kyoto 603-8555, Japan \\ hidehiko@cc.kyoto-su.ac.jp \\ ${ }^{2}$ Kobe Sogo Sokki Co. Ltd. \\ 4-3-8 Kitanagase-dori, Chuo-ku, Kobe, Hyogo, 650-0012, Japan \\ ryo@sogosokki.co.jp
}

\begin{abstract}
It is well-known as Fitts' law that the time for a user to point a target on a GUI screen can be modeled as a linear function of "index of difficulty (ID)". The authors investigate whether the ID formulation is appropriate independently of device screen sizes. Result of our experiment revealed that the ID formulation may not consistently capture actual difficulty: users' pointing performances were not consistent among pointing target variations of which index of difficulty are consistent. The term $\mathrm{A} / \mathrm{W}$ may not be appropriate because the term causes the observed inconsistency.
\end{abstract}

Keywords: usability, Fitts' law, touch user interface, small screen, smart phone, throughput, error rate.

\section{Introduction}

It is well-known as Fitts' law that the time for a user to point a target can be modeled as a linear function of "index of difficulty (ID)", where ID is formulated as a function of the target size and distance $[1,2]$.

$$
\begin{gathered}
\mathrm{t}=\mathrm{a}+\mathrm{b} * \mathrm{ID} . \\
\mathrm{ID}=\log _{2}(\mathrm{~A} / \mathrm{W}+1) .
\end{gathered}
$$

In Eqs. (1-2), $\mathrm{t}$ is the pointing time, $\mathrm{A}$ is the amplitude (distance) to the target, $\mathrm{W}$ is the target size and $\mathrm{a}, \mathrm{b}$ are constant that depend on experiment conditions. ID is larger as $\mathrm{A}$ is larger and/or $\mathrm{W}$ is smaller. Values of $\mathrm{a}$ and $\mathrm{b}$ in Eq. (1) are determined by sampling $(\mathrm{A}, \mathrm{W}, \mathrm{t})$ data and applying the linear regression analysis to the data. Eq. (2) shows that ID values are the same for $(\mathrm{A}, \mathrm{W})$ and $(\mathrm{nA}, \mathrm{nW})$ where $\mathrm{n}>0$.

This research is motivated by recent smart phones that employ touch UIs. Compared with other touch screen devices such as tablet PCs, mobile phones have smaller screens so that widgets on mobile phone screens are likely to be smaller. Widgets can be designed for devices with various screen sizes so that theoretical ID values in Eq. (2) are consistent among the devices: larger/smaller sizes\&distances for 
larger/smaller screens. If ID in Eq. (2) is an appropriate index of actual pointing difficulty independently of screen sizes, users' pointing performances on the same device are consistent among widget designs $(\mathrm{A}, \mathrm{W})$ and $(\mathrm{nA}, \mathrm{nW})$ : note that $\mathrm{a}, \mathrm{b}$ in Eq. (1) are constant (independent to ID) so that $a, b$ must be the same for two data sets sampled with the two widget designs $(\mathrm{A}, \mathrm{W})$ and $(\mathrm{nA}, \mathrm{nW})$. The aim of this research is to investigate whether the above is true: appropriateness of the ID formulation in Eq. (2) is evaluated from the viewpoint of dependency on screen sizes, by experiments with participants.

Limitations of Fitts' law have been researched and extensions have been proposed. For example, MacKenzie et al. [3] proposed an extension for 2D pointing tasks. Our research aims at investigating possible limitations on screen sizes. Beside, a related research was previously reported by Oehl et al.[4]. They investigated how display size influenced pointing performances on a touch UI and reported that in large displays a fast and comparably accurate execution was chosen in contrast to a very inaccurate and time-consuming style in small displays. In their research the size of small screen was 6.5", and only a large screen touch UI device was utilized for user experiments: screen sizes were controlled by means of software program as virtual screens on the device display. In our research, the size of small screen is less than 3", and a commercial smaller-screen mobile device is utilized.

\section{Experiments}

\subsection{Test Tasks}

Participants were asked to point targets on a screen. A test task consisted of pointing two rectangle targets (target 1,2 ) in a predefined order. An "attempt" was the two successive pointings of target 1 and 2, and a test task consisted of a predefined number set of the attempts. For each combination of experiment conditions, each participant was asked to perform a predefined set of the tasks. The pointing operations were logged for later analyses of pointing speed and accuracy.

\subsection{Conditions}

Devices. Three commercial devices were used in our experiment: two tablet PCs and a PDA which have a $\{10.2 ", 6.0 ", 2.8 "\}$ touch screen respectively. The PDA was selected because several recent smart phones have such small touch screens (i.e., the PDA was used as a substitute for the recent smart phones). Screen sizes of the devices were relatively larger/middle/smaller. In this paper, these devices are denoted as devices $\mathrm{L} / \mathrm{M} / \mathrm{S}$ respectively. Participants performed test tasks by using a stylus attached to each of the three devices ${ }^{1}$.

Target Sizes \& Distances. For each of the three devices, two sets of targets were designed so that ID values in Eq. (2) were consistent between the two sets. Targets in

${ }^{1}$ Differences in stylus designs may affect pointing performances as reported by Ren et al.[5]. It is assumed in our research that the stylus attached to each device is designed optimal for the device so that the stylus contributes to achieve better performances on the device than other styluses. 
one of the two sets were designed with smaller sizes and distances, and those in the other were designed with larger ones. Specific designs of the two target sets are described later. In this paper, these two target sets are denoted as targets $\mathrm{L} / \mathrm{S}$ respectively.

Errors. Pointing speed and accuracy are usually a tradeoff [6]. Participants performed tasks under each of two error conditions: errors acceptable or not. In a test task where errors were acceptable, a participant could continue the task even if s/he made an error (misspointing), and the task was complete when the count of no-error attempts reached to a predefined number. In a condition where errors were not acceptable, a test task was cancelled by misspointing and the task was retried until the count of noerror attempts reached to a predefined number. The error condition was told to each participant before performing each task: s/he had to try a task more carefully in the errors-not-acceptable condition.

\subsection{Pointing Target Designs}

Table 1 shows the design of target sizes and distances. Values for the device $\{\mathrm{M}, \mathrm{L}\}$ were determined as [values for the device $S$ ] * [the ratio of screen sizes, i.e., 6.0/2.8 for the device $\mathrm{M}$ and $10.2 / 2.8$ for the device $\mathrm{L}]$. ID values were designed to range in $[2.00,3.50]$ consistently among the devices $\{\mathrm{S}, \mathrm{M}, \mathrm{L}\}$ and the targets $\{\mathrm{S}, \mathrm{L}\}$. The size of target 1 was fixed to $6.0 \mathrm{~mm}$, empirically found to be easy enough to point first, for all conditions. Positions of targets 1 and 2 were randomly determined for each attempt under the following two constraints.

- All area of both targets were inside the device screen.

- Distance between center points of the two targets was a predefined value.

Fig. 1 shows a screenshot of targets 1 and 2 for the device $M$ and the targets L. The targets 1 and 2 are the black and white rectangles respectively (the target colors were consistent for all the devices). The two targets were shown at the same time, and each participant was asked to find both targets before s/he pointed the target 1 . This was because visual search time should not be included in the pointing time interval. After an attempt of pointing targets 1 and 2, new targets were shown for the next attempt.

\subsection{Methods of Experiments}

Condition combinations were 12 in total: $\{$ the devices $\mathrm{S}, \mathrm{M}, \mathrm{L}\} *\{$ the $\operatorname{targets} \mathrm{S}, \mathrm{L}\} *$ \{errors "acceptable", "not acceptable"\}. Each participant was asked to perform four trials of a task under each of the 12 condition combinations.

The number of attempts in a task trial was 11 (of which ID=2.00-3.50 shown in Table 1) for the errors "not acceptable" condition: none of the 11 attempts had to be an error. For the errors "acceptable" condition, a task trial included 11 successful attempts for the 11 IDs respectively in Table 1 and $0+$ error attempts.

Each participant first performed a training task trial under each of the 12 condition combinations (thus, 12 training trials), and then performed tasks in a random order of the 12 condition combinations. The order of the 11 IDs in a trial was also randomized for each trial. 
Table 1. Target sizes and distances

\begin{tabular}{|c|c|c|c|c|c|c|c|c|c|c|c|c|}
\hline & \multicolumn{4}{|c|}{ Device $\mathrm{S}$} & \multicolumn{4}{|c|}{ Device M } & \multicolumn{4}{|c|}{ Device L } \\
\hline & \multicolumn{2}{|c|}{ Targets S } & \multicolumn{2}{|c|}{ Targets L } & \multicolumn{2}{|c|}{ Targets S } & \multicolumn{2}{|c|}{ Targets L } & \multicolumn{2}{|c|}{ Targets S } & \multicolumn{2}{|c|}{ Targets L } \\
\hline ID & $\mathrm{W}$ & $\mathrm{A}$ & $\mathrm{W}$ & $\mathrm{A}$ & $\mathrm{W}$ & $\mathrm{A}$ & $\mathrm{W}$ & $\mathrm{A}$ & $\mathrm{W}$ & $\mathrm{A}$ & $\mathrm{W}$ & $\mathrm{A}$ \\
\hline 2.00 & 4.00 & 12.00 & 12.00 & 36.00 & 8.53 & 25.60 & 25.60 & 76.80 & 14.61 & 43.82 & 43.82 & 131.45 \\
\hline 2.15 & 3.80 & 13.07 & 11.40 & 39.20 & 8.11 & 27.87 & 24.32 & 83.62 & 13.88 & 47.71 & 41.63 & 143.12 \\
\hline 2.30 & 3.60 & 14.13 & 10.80 & 42.39 & 7.68 & 30.14 & 23.04 & 90.42 & 13.15 & 51.59 & 39.44 & 154.77 \\
\hline 2.45 & 3.40 & 15.18 & 10.20 & 45.53 & 7.25 & 32.38 & 21.76 & 97.14 & 12.41 & 55.42 & 37.24 & 166.26 \\
\hline 2.60 & 3.20 & 16.20 & 9.60 & 48.60 & 6.83 & 34.56 & 20.48 & 103.69 & 11.68 & 59.16 & 35.05 & 177.47 \\
\hline 2.75 & 3.00 & 17.18 & 9.00 & 51.54 & 6.40 & 36.65 & 19.20 & 109.96 & 10.95 & 62.74 & 32.86 & 188.21 \\
\hline 2.90 & 2.80 & 18.10 & 8.40 & 54.30 & 5.97 & 38.61 & 17.92 & 115.84 & 10.22 & 66.09 & 30.67 & 198.27 \\
\hline 3.05 & 2.60 & 18.93 & 7.80 & 56.80 & 5.55 & 40.39 & 16.64 & 121.17 & 9.49 & 69.13 & 28.48 & 207.40 \\
\hline 3.20 & 2.40 & 19.66 & 7.20 & 58.97 & 5.12 & 41.93 & 15.36 & 125.79 & 8.76 & 71.77 & 26.29 & 215.31 \\
\hline 3.35 & 2.20 & 20.23 & 6.60 & 60.70 & 4.69 & 43.16 & 14.08 & 129.49 & 8.03 & 73.88 & 24.10 & 221.63 \\
\hline 3.50 & 2.00 & 20.63 & 6.00 & 61.88 & 4.27 & 44.01 & 12.80 & 132.02 & 7.30 & 75.32 & 21.91 & 225.96 \\
\hline
\end{tabular}

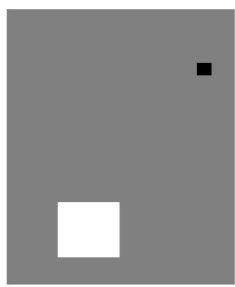

Fig. 1. Screenshot for target pointing tasks

\subsection{Participants}

Twelve subjects participated in the experiment, but 3 of the 12 subjects could for the devices $\mathrm{S}$ and $\mathrm{L}$ only due to the experiment schedule. Thus, users' pointing log data set $(\mathrm{A}, \mathrm{W}, \mathrm{t})$ were collected with 12 subjects for the devices $\mathrm{S}$ and $\mathrm{L}$ but 9 subjects for the device $\mathrm{M}$. The 12 participants were university graduate or undergraduate students. They were all novices in using devices with touch-by-stylus UIs, but they had no trouble in performing test tasks after the 12 training trials.

\subsection{Logging Pointing Operations}

The following data was recorded for each pointing (each tap by a stylus) into log files.

- Target: 1 or 2

- Target position: $(\mathrm{x}, \mathrm{y})$ values

- Target width and height: pixels

- Tapped position: $(\mathrm{x}, \mathrm{y})$ values

- Tap time: msec

- Error: Yes or No 
The tapped position and the tap time were logged when the stylus was land on the screen, and the pointing was judged as an error or not based on the tapped position. No attempt was observed for which the stylus was landed on the target 1 , moved into the target 2 and left off.

\section{Data Analyses and Findings}

Pointing speed and accuracy were measured by throughput [7] and error rate respectively. In this research, $t$ is the interval from the target 1 tap time to the target 2 tap time, A is the Euclid distance between the tapped points for targets 1 and 2, and W is the target width (= height). Throughput is defined as ID/t in Eqs. (1-2) [7]. (ID, t) could be observed for each attempt, so a throughput value could also be obtained for each attempt. To measure pointing accuracy, error rate was defined.

Error rate $=(\#$ error attempts in a task trial $) /(\#$ total attempts in the trial $)$

Error rate could be calculated for only the condition "error"="acceptable" because the data under the condition "error"="not acceptable" didn't include any error attempt (if an error was occurred in a trial under the condition "error"="not acceptable", the trial was cancelled and retried).

Mean and standard deviation (SD) values of the throughput and the error rate were calculated to compare user performances on targets $\mathrm{S}$ to those on targets $\mathrm{L}$. Throughput mean and SD values were calculated from the data of $\{\operatorname{tp}(s, t, a(s, t))\}$ for all of the subjects, the task trials and the attempts in a task: $\operatorname{tp}(\mathrm{s}, \mathrm{t}, \mathrm{a}(\mathrm{s}, \mathrm{t}))$ denotes the throughput value for the s-th subject, t-th task and the a(s,t)-th attempt in the t-th task by the s-th subject. Error rate mean and SD values were calculated from the data of $\{\operatorname{er}(\mathrm{s}, \mathrm{t})\}$ for all of the subject and the task trials: er(s, t) denotes the error rate value for the s-th subject and the t-th task.

In addition, it was tested by t-test whether there was a significant difference between population mean values of throughput and error rate for two conditions.

It should be noted that error attempts were included in the data under the condition "error"="acceptable". Error attempts might be faster (of larger throughput values) than successful attempts. In the following of this paper, throughput values were calculated with both of successful and error attempt data.

Table 2 shows mean and SD values of the throughput, and Table 3 shows those of the error rate. Tables $4 \& 5$ show t-test results for throughput and error rate respectively. In Tables $4 \& 5$, “**”-marked t-scores are those with $\mathrm{p}<0.01$, and nonmarked t-scores are those with $\mathrm{p}>0.05$.

Table 2. Mean and SD values of throughput (bit/sec)

\begin{tabular}{llrrrrrr}
\hline & & \multicolumn{2}{c}{ Device S } & \multicolumn{2}{c}{ Device M } & \multicolumn{2}{c}{ Device L } \\
\cline { 3 - 8 } & & Targets S & Targets L & Targets S & Targets L & Targets S & Targets L \\
\hline \multirow{2}{*}{ Acceptable } & Mean & 5.73 & 5.73 & 5.86 & 5.76 & 5.52 & 4.76 \\
& SD & 1.37 & 1.14 & 1.30 & 1.80 & 1.34 & 0.87 \\
\cline { 2 - 7 } Not acceptable & Mean & 5.15 & 5.57 & 5.69 & 5.63 & 5.32 & 4.60 \\
& SD & 1.20 & 1.21 & 1.30 & 1.78 & 1.23 & 0.97 \\
\hline
\end{tabular}


Table 3. Mean and SD values of error rate (\%)

\begin{tabular}{llrrrrrr}
\hline & \multicolumn{2}{c}{ Device S } & \multicolumn{2}{c}{ Device M } & \multicolumn{2}{c}{ Device L } \\
\cline { 2 - 7 } & & Targets S & Targets L & Targets S & Targets L & Targets S & Targets L \\
\hline \multirow{2}{*}{ Acceptable } & Mean & 11.23 & 0.52 & 0.93 & 0.69 & 1.56 & 0.52 \\
& SD & 10.35 & 2.04 & 2.66 & 2.34 & 3.29 & 2.04 \\
\hline
\end{tabular}

Table 4. T-test for throughput

\begin{tabular}{ccccccc}
\hline & \multicolumn{2}{c}{ Device $\mathrm{S}$} & \multicolumn{2}{c}{ Device M } & \multicolumn{2}{c}{ Device L } \\
\cline { 2 - 7 } & Acceptable & $\begin{array}{c}\text { Not } \\
\text { acceptable }\end{array}$ & Acceptable & $\begin{array}{c}\text { Not } \\
\text { acceptable }\end{array}$ & Acceptable & $\begin{array}{c}\text { Not } \\
\text { acceptable }\end{array}$ \\
\hline Targets S/L & $\mathrm{t}=3.65 * 10^{-3}$ & $\mathrm{t}=-5.74 * *$ & $\mathrm{t}=0.875$ & $\mathrm{t}=0.514$ & $\mathrm{t}=11.04 * *$ & $\mathrm{t}=10.66^{* *}$ \\
\hline
\end{tabular}

Table 5. T-test for error rate

\begin{tabular}{llll}
\hline & \multicolumn{3}{c}{ Acceptable } \\
\cline { 2 - 4 } & Device S & Device M & Device L \\
\hline Targets S/L & $\mathrm{t}=7.03^{* *}$ & $\mathrm{t}=0.393$ & $\mathrm{t}=1.87$ \\
\hline
\end{tabular}

These tables revealed the followings.

- On the device L participants could point targets S significantly faster than targets L, but on the devices S\&M they couldn't. Instead, on the device S, they could point targets L significantly faster than targets S under the condition "error"="not acceptable". This result indicates that, even though ID values by (2) are designed consistently among targets S\&L, users' pointing speeds will not be consistent: faster for larger/smaller size\&distance widgets on smaller/larger screen devices, respectively.

- On the devices M\&L no significant difference was observed in the pointing accuracy among targets $\mathrm{S} \& \mathrm{~L}$, but on the device $\mathrm{S}$ participants could point targets $\mathrm{L}$ significantly more accurately than targets $\mathrm{S}$. This result indicates that, even though ID values by (2) are designed consistently among targets S\&L, users' pointing accuracies will not be consistent too: more accurate for larger size\&distance widgets on smaller screen devices.

Thus, it is found that the ID definition in (2) may not consistently capture actual pointing difficulty among target designs. The result of our experiment shows that, on a smaller/larger screen, targets with smaller/larger sizes\&distances are actually more difficult to point than those with larger/smaller ones. A/W in (2) is not appropriate in terms of screen size variations because the term caused the observed inconsistency.

\section{Conclusion}

Index of difficulty formulation in Fitts' law was evaluated from the viewpoint of consistency in widget size\&distance design variations. It was found that ID in (2) may 
not appropriately capture actual difficulty: user performances on the same device were not consistent among target designs (A, W) and $(\mathrm{nA}, \mathrm{nW})$. Further research is necessary to investigate better formulation of ID.

\section{References}

1. Fitts, P.M.: The Information Capacity of the Human Motor System in Controlling the Amplitude of Movement. Journal of Experimental Psychology 47(6), 381-391 (1954)

2. MacKenzie, I.S.: Fitts's Law as a Research and Design Tool in Human-Computer Interaction. Human-Computer Interaction 7, 91-139 (1992)

3. MacKenzie, I.S., Buxton, W.: Extending Fitts' Law to Two-dimensional Tasks. In: Proc. of ACM Conf. on Human Factors in Computing Systems (CHI 1992), pp. 219-226 (1992)

4. Oehl, M., Sutter, C., Ziefle, M.: Considerations on Efficient Touch Interfaces - How Display Size Influences the Performance in an Applied Pointing Task. In: Smith, M.J., Salvendy, G. (eds.) HCII 2007. LNCS, vol. 4557, pp. 136-143. Springer, Heidelberg (2007)

5. Ren, X., Mizobuchi, S.: Investigating the Usability of the Stylus Pen on Handheld Devices. In: Proceedings of The Fourth Annual Workshop on HCI Research in MIS, pp. 30-34 (2005)

6. Plamondon, R., Alimi, A.M.: Speed/Accuracy Trade-offs in Target-Directed Movements. Behavioral and Brain Sciences 20(2), 279-349 (1997)

7. ISO 9241, Ergonomic Requirements for Office Work with Visual Display Terminals (VDTs) - Part 9: Requirements for Non-Keyboard Input Devices (2000) 